\title{
Some Luminescence Properties of the Laser Dye Stilbene 3
}

\author{
R. K. Bauer, A. Balter, A. Kowalczyk \\ Institute of Physics, Nicolaus Copernicus University, Grudziądzka 5, 87-100 Torun, Poland \\ and
}

Ch. Jung

Sektion Chemie, Humboldt Universität, 108 Berlin, Bunsenstr. 1, GDR

Z. Naturforsch. 35a, 1319-1324 (1980); received October 6, 1980

\begin{abstract}
Absorption and emission spectra of stilbene 3 (Na-bis-ortho-stilbene-sulphonate) were measured and discussed on the basis of quantum-chemical calculations. The decay of the polarized fluorescence was investigated, yielding information about the rotational behaviour of stilbene 3 in solutions.
\end{abstract}

\section{Introduction}

Dye laser materials should be resistent to photodecomposition and should have a high quantum yield and good thermal stability. In the blue-violet lasing region, scintillator and coumarin dyes are often used. A new class of laser dyes in this spectral region are the bis-styryl compounds $[1,2,3]$. Stilbene 3 (Na-bis-ortho-stilbene sulphonate) in particular is a highly efficient photostable dye, soluble in water and alcohols. Strong absorption in the wavelength region of $300-380 \mathrm{~nm}$ makes this dye suitable for excitation which $\mathrm{N}_{2}$-pulse and Arcw lasers.

With this dye, we obtained tunable laser action generating single spikes of less than $450 \mathrm{ps}$ fwhm duration in ethylene glycol-methanol solutions excited with a subnanosecond compact TEA $\mathrm{N}_{2}$ laser [4] of only $10 \mu \mathrm{J}$ pulse energy but $200 \mathrm{~Hz}$ repetition rate. It should be emphasized that no laser action could be obtained under these conditions for rhodamine $6 \mathrm{G}$ or 4 -methylumbelliferone solutions. These favourable properties of stilbene 2 motivated us to study its spectral characteristics along with the decay and polarization of its fluorescence, as well as to perform some relevant quantumchemical calculations for this molecule.

\section{Quantum-Chemical Calculations}

We applied the PPP method with the MatagaNishimoto integral approximation. In the CI cal-

Reprint requests to Prof. R. K. Bauer, Institute of Physics, Nicolaus Copernicus University, Grudziądzka 5, 87-100 Toruń, Poland. culations, all those configurations which are symmetry allowed in the first singlet excited state, were included. The geometrical model shown in Fig. 1 was used. The stilbene-like parts were taken to be planar with bond lengths $0.14 \mathrm{~nm}$ and $0.148 \mathrm{~nm}$ in the benzene rings and between the ethylene carbon atoms and benzene rings, respectively. We assumed the length of the ethylenic bonds to be $0.135 \mathrm{~nm}$ and all angles $120^{\circ}$. For a justification of these assumptions see Ref. [5]. In contrast to [3], the trans-configuration of the whole molecule was assumed because of the repelling interaction between the two sulphonate groups, which carry a negative charge on their oxygen atoms.

The length of the central bond between the two stilbene-like parts was assumed to be $0.15 \mathrm{~nm}$, and two values for the twisting angle $\Theta$ around this

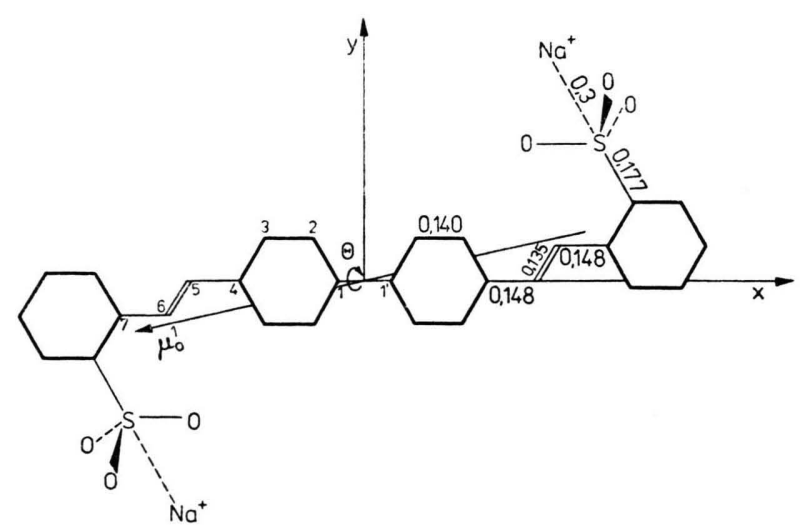

Fig. 1. Geometrical model of stilbene 3 with $\Theta=0^{\circ}$. Bond lengths are given in $\mathrm{nm}$. 
bond were considered: $45^{\circ}$ for the ground state conformation and $0^{\circ}$ for the first excited state, by analogy with biphenyl [6]. Geometry-dependent resonance integrals $\beta_{\text {rs }}$ between carbon atoms were used as follows:

$$
\begin{aligned}
\beta_{\mathrm{rs}}(\Theta) & =\beta_{0} \cos \Theta, \\
\beta_{\mathrm{rs}}\left(R_{\mathrm{rs}}\right) & =\beta_{0} \exp \left(-18.62 R_{\mathrm{rs}}\right)+2.597,
\end{aligned}
$$

with $R_{\mathrm{rs}}$ in $\mathrm{nm}$. Provision was made for the inclusion of $\mathrm{p} \Pi-\mathrm{d} \Pi$ interactions within the tetrahedrally hybridized sulphonate group (see for example [7]): $d_{z^{2}}$ and $d_{x^{2}-y^{2}}$ orbitals are especially suitable for $\Pi$ interactions, which were therefore included in the PPP calculations taking the sulphur atom to be located in the stilbene plane.

It should be mentioned that stilbene 3 is a dianion and $\mathrm{Na}^{+}$does not form ion pairs with it, as indicated by the the high conductivity of stilbene 3 water and glycerol solutions. Nevertheless it is important to take the interaction of these charges into account. If this interaction is neglected, much too low excitation energies are obtained. The strong influence of the position of positive charges with respect to the sulphur atom can be interpreted as a field effect operating differently in the ground and excited states. This difference is connected with a slight charge shift from the oxygen atoms to sulphur and carbon atoms in the first excited state, although the charge of the whole sulphonate group remains constant. The results presented in this work were calculated with two positive charges placed about $0.3 \mathrm{~nm}$ from the sulphur atoms, in line with the C-S bonds. Table 1 contains the parameters used in our calculations. For comparison, calculations using the same parametrization were performed for Na-

Table 1. Parameters for the PPP calculations.

\begin{tabular}{lccccc}
\hline$\Pi$-atom X & $I_{\mathrm{p}}(\mathrm{X})^{\mathrm{a}}$ & $N_{\Pi^{\mathrm{b}}}$ & $\gamma_{\mathrm{XX}}^{\mathrm{c}}$ & $K_{\mathrm{CX}} \mathrm{d}^{\mathrm{d}}$ & $r_{\mathrm{CX}}[\mathrm{nm}]$ \\
\hline$-\mathrm{C}=$ & 9.84 & 1 & 10.53 & 1.0 & see text \\
$-\mathrm{O}$ & 17.70 & 1 & 15.23 & 1.1 & 0.144 \\
$\begin{array}{l}\text { I } \\
\text { I }\end{array}$ & 1.60 & 1 & 1.80 & 0.7 & 0.177 \\
\hline
\end{tabular}

a valence state ionization potential in $\mathrm{eV}$ (for the $\mathrm{CH}_{3}$ radical the value for the carbon atom is assumed).

b number of $I$ electrons per atomic orbital.

c one center two electron integral in $\mathrm{eV}$.

d factor for $\beta_{\mathrm{CX}}=\beta_{0} K_{\mathrm{CX}}$ with $\beta_{0}=2.318 \mathrm{eV}$.
Table 2. Calculated properties of the ground and first excited singlet states of stilbene 3, Na-ortho-stilbene

\begin{tabular}{|c|c|c|c|c|}
\hline \multicolumn{3}{|c|}{$\begin{array}{l}\text { Stilbene } 3 \\
\text { twisting angle }\end{array}$} & \multirow{2}{*}{$\begin{array}{l}\text { Na-ortho- } \\
\text { stilbene } \\
\text { sulphonate }\end{array}$} & \multirow[t]{2}{*}{ Stilbene } \\
\hline$\Theta$ & $0^{\circ}$ & $45^{\circ}$ & & \\
\hline$\Delta E / f^{\mathrm{a}}$ & $30.11 / 2.25$ & $31.57 / 2.21$ & $32.83 / 1.04$ & $4 \quad 33.65 / 1.14$ \\
\hline$\left|\vec{\mu}_{0}^{1}\right|^{\mathrm{b}}$ & 2.63 & 2.54 & 1.71 & 1.71 \\
\hline$\Varangle\left(\mu_{0}{ }^{1}, x\right)^{\mathrm{e}}$ & 167 & 166 & 165 & 167 \\
\hline$\Varangle\left(\mu_{0}^{1}, y\right)$ & 103 & 103 & 105 & 103 \\
\hline$\Varangle\left(\mu_{0}^{1}, z\right)$ & 90 & 85 & 90 & 90 \\
\hline$Q\left(-\mathrm{SO}_{3}\right)^{\mathrm{c}}$ & -1.00 & -1.00 & -1.03 & - \\
\hline$Q^{*}\left(-\mathrm{SO}_{3}\right)$ & -1.00 & -1.00 & -0.99 & - \\
\hline$P_{1,1}, \mathrm{a}$ & 0.28 & 0.20 & - & - \\
\hline$P_{1,1^{\prime}}^{*}$ & 0.40 & 0.30 & - & - \\
\hline$P_{6,7}$ & 0.30 & 0.30 & 0.31 & 0.37 \\
\hline$P_{6,7}^{*}$ & 0.38 & 0.40 & 0.53 & 0.58 \\
\hline$P_{5,6}$ & 0.90 & 0.90 & 0.90 & 0.86 \\
\hline$P_{5,6}^{*}$ & 0.77 & 0.75 & 0.58 & 0.50 \\
\hline
\end{tabular}
sulphonate and stilbene.

a $S_{0}-S_{1}$ transition energy $\left[10^{3} \mathrm{~cm}^{-1}\right] /$ oscillator strength.

b transition moment [Debye].

c charge of sulphonate group.

d bond orders in the ground and excited (*) state (for numbering of atoms see Figure 1).

e for the coordinate system $x y z$ ef. Figure 1.

ortho-stilbene-sulphonate and other stilbenes [8]. The results of our calculations concerning the energies and transition moments are shown in Table 2.

Some interesting features of stilbene 3 are evident from the results presented in Table 2 . Thus, if we were treating stilbene 3 like a dimer of two nonoverlapping Na-ortho-stilbene-sulphonate monomers $M$, we should obtain a doubled value of the transition moment to the excited state

$$
1 \Psi^{*}=\frac{1}{\sqrt{ } 2}\left(\Psi_{\mathrm{M} 1} \Psi_{\mathrm{M} 2}^{*}-\Psi_{\mathrm{M} 1}^{*} \Psi_{\mathrm{M} 2}\right),
$$

because of the tail to tail arrangement of the two monomer transition dipoles [9].

The somewhat lower value actually obtained is connected with the overlap of the two monomer electronic wavefunctions. It should be mentioned that the first excited state is characterized by a HOMO $\rightarrow$ LUMO orbital transition and leads to a more quinoidal structure, as in the case of stilbene. The changes of bond orders confirm this conclusion. An especially interesting feature of stilbene 3 is the strengthening of the central bond 1-1 in the excited state.

This supports the assumed analogy with biphenyl. 


\section{Absorption and Emission Spectra}

A sample of stilbene 3, received from Dr. R. Raue, was used without further purification in the solvents ethylene glycol, glycerol ( $5 \%$ aqueous), water and methanol. Special care was taken to avoid any fluorescence of the solvente themselves. At the concentration $2 \times 10^{-5} \mathrm{M}$ the optical density did not exceed 0.2. In the absorption spectra of stilbene 3 in water and ethylene glycol (Fig. 2.) one observes a pronounced shift between these two absorption curves. The absorption spectra measured in water and methanol are practically the same, as is also the case for glycerol and ethylene glycol. The observed solvent shift cannot be explained by different dielectric influences. The only parameter of the solvents apparently correlated with the shift is the viscosity, but we cannot provide any explanation of this phenomenon.

The calculated electronic transition energies and relative oscillator strengths are also compared with the experimental spectra in Figure 2. For chargesulphur atom separations within the range of 0.22 to $0.35 \mathrm{~nm}$, the three absorption bands agree qualitatively with the calculated allowed electronic transitions. The agreement for these transitions is best at about $0.3 \mathrm{~nm}$. The other properties did not change significantly within this range.

The emission spectra were measured in glycerol, in ethylene glycol and in a 1:1 water-ethylene glycol mixture at different temperatures and excitation wavelengths. A "Spectrim" (CTi-Cyrogenics) sample cooler was used to attain the low temperatures. Some of the emission spectra obtained are also shown in Figure 2. It is evident that the absorption and emission spectral overlap is small. A characteristic feature of the emission spectra is the pronounced vibrational structure contrasting with the structureless absorption profiles. This phenomenon may be understood in terms of the angular orientation of the two stilbene-sulphonate moieties connected by a biphenyl-like bond. There is a strong analogy to the case of biphenyl [10]. As is well known, this molecule is planar in its first excited state but twisted in the ground state by $42^{\circ}$ [11].

The emission spectra of stilbene 3 in glycerol for different temperatures and excitation wavelengths are shown in Figure 3. At $340 \mathrm{~K}$ the emission spectra are red-shifted, practically independent of the excitation wavelength and broader than the spectra measured at $80 \mathrm{~K}$. At this lower temperature the

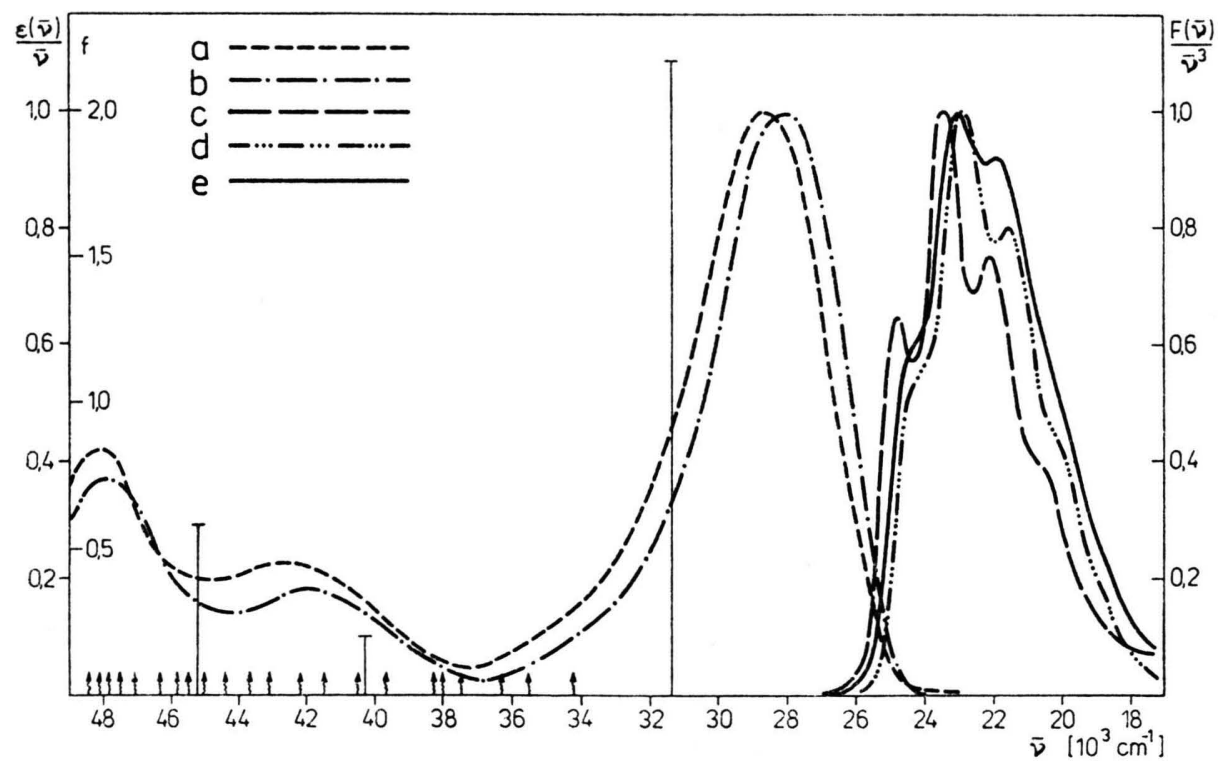

Fig. 2. Normalized absorption and emission spectra of stilbene 3 under various conditions. Absorption spectra for (a) water and methanol, and (b) ethylene glycol and glycerol solutions at $293 \mathrm{~K}$. Fluorescence spectra for (c) $1: 1$ mixed water and ethylene glycol solutions at (d) $20 \mathrm{~K}$ and (e) $293 \mathrm{~K}$. Excitation wavelength: $365 \mathrm{~nm}$. The vertical bars represent the calculated transition energies and oscillator strengths $f$ for a charge-sulphur atom separation of $0.2 \mathrm{~nm}$. The arrows indicate transitions forbidden for the plane geometry, slightly allowed for the twisted one. 


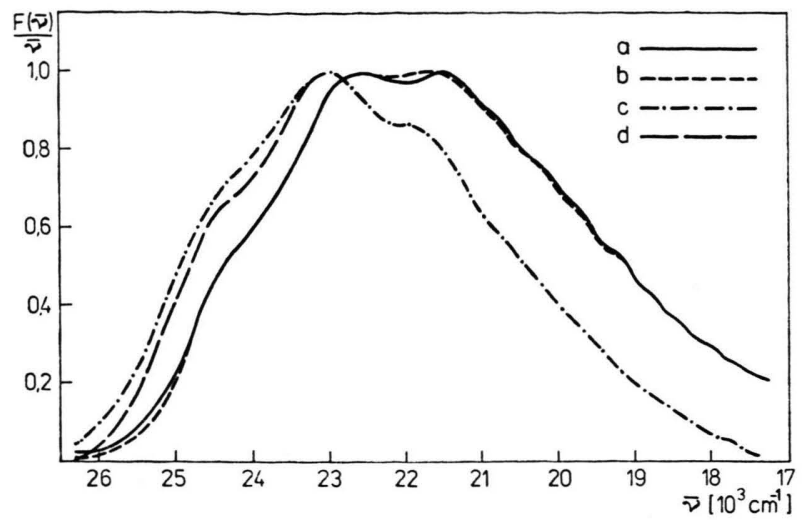

Fig. 3. Fluorescence spectra of stilbene 3 in glycerol at $340 \mathrm{~K}(\mathrm{a}, \mathrm{b})$ and $80 \mathrm{~K}(\mathrm{~d}, \mathrm{~d})$. Excitation wavelengths were $313 \mathrm{~nm}(\mathrm{a}, \mathrm{c})$ and $365 \mathrm{~nm}(\mathrm{~b}, \mathrm{~d})$.

emission excited at $313 \mathrm{~nm}$ is broader at the blue edge and less structured in comparison with the emission excited at $365 \mathrm{~nm}$. Besides the observed spectral shift, the temperature change also leads to a change in the relative emission intensities of the vibrational bands. Such a change in the structure of the emission spectra, superimposed upon the spectral shift, indicates that the latter cannot be explained only on the basis of the Onsager-Böttcher theory [12]. We would like to interpret the temperature induced change of the vibrational structure in the following way: the $\mathrm{C}-\mathrm{C}$ stretching potential in the ground state reaches its minimum at a considerably shorter $\mathrm{C}-\mathrm{C}$ bond length for the twisted geometry compared with the non-twisted one (repulsion between the ortho-hydrogens [10]. In the excited state, due to the strengthening of the biphenyllike $\mathrm{C}-\mathrm{C} \Pi$-bond which compensates the repulsion between hydrogen atoms, the twisting potential becomes more flat, and therefore the dependence of the $\mathrm{C}-\mathrm{C}$ stretching potential minimum on the twisting angle should be small.

At $340 \mathrm{~K}$ the relaxation along the twisting angle coordinate enables the vertical emissive transition from the minimum of the twisting potential curve. This relaxation is hindered at $80 \mathrm{~K}$ and the transition to the ground state takes place from higher twisting levels to different values of the $\mathrm{C}-\mathrm{C}$ stretching coordinate in the ground state, i.e., to other vibrational levels. From the above considerations it follows that different vibrational intensities are possible at different temperatures. This model also accounts for the bathochromic shift accompanying the temperature increase.
The excitation wavelength dependence of the emission profile at the blue edge supports the concept of Van Metter and Knox [13]. Basing their treatment on the idea of inhomogeneous broadening introduced by Mazurenko [14], they dealt quantitatively with the excitation wavelength dependence of spectra. According to their theory, such a dependence of the emission spectra should occur if the average lifetime $\tau_{\mathrm{s}}$ of the fluctuations in the environment of the solute molecule is longer than the mean fluorescence lifetime $\tau_{\mathrm{f}}$. At $80 \mathrm{~K}$ one can safely estimate that $\tau_{\mathrm{s}}$ must be orders of magnitude longer than $\tau_{\mathrm{f}}$. This is not so at $340 \mathrm{~K}$, where the inequality $\tau_{\mathrm{s}} \gg \tau_{\mathrm{f}}$ no longer holds and the excitation wavelength dependence of the emission spectra practically disappears.

\section{Decay and Polarization of Emission}

Due to Brownian rotations of the dye molecule together with its solvation shell, the parallel and perpendicular polarized components of the fluorescence intensity do not decay monoexponentially and their mean decay times are different. As was first shown by Jabłoński [15], the decay of these components is governed by the equations:

$$
\begin{aligned}
I^{\|}(t) & =\frac{I_{0}}{3}\left(1+2 r_{0} e^{-\varphi t}\right) e^{-t / \tau}, \\
I^{\perp}(t) & =\frac{I_{0}}{3}\left(1-r_{0} e^{-\varphi t}\right) e^{-t / \tau},
\end{aligned}
$$

if one assumes that both the molecular emission and the emission anisotropy (EA) $r(t)$, decay monoexponentially. In Eq. (1), $r_{0}$ denotes the limiting $\mathrm{EA}$, i.e. the value observed when the rotational depolarization is negligible, $\varphi$ is the reciprocal of the rotational correlation time and $\tau$ is the mean lifetime of the excited state.

The assumption of the monoexponential decay of the EA, $r(t)=r_{0} \exp (-\varphi t)$ is justified if the dye molecule with its solvation shell rotates isotropically, i.e. if it is effectively spherical or if the absorption and/or emission dipole moment is parallel with the symmetry axis of an effective ellipsoid of revolution. Based upon Eq. (1), Jabłoński derived expressions for the mean decay times of the polarized fluorescence components. If these decay times $\tau \|$ and $\tau^{\perp}$ are measured as a function of temperature $T$ and/or viscosity $\eta$ together with the mean EA $\langle r\rangle$, one is able to calculate the value of $r_{0}$ and 
$\varphi=k T / \eta V$ and finally the effective volume of the dye with its solvation shell $V$ [16]. The decay times of the polarized components and the EA of the fluorescence of stilbene 3 dissolved in glycerolmethanol mixtures were measured by means of a phase-fluorometer and compensation polarimeter, respectively. The viscosity of these mixtures was measured using the Hoeppler viscometer.

The results of these measurements are presented in Table 3 and Figure 4 . The quite accurate fit between calculated and measured values of $\tau^{\prime \prime} / \tau$ and $\tau^{\perp} / \tau$ means that the assumption of an exponential decay of the EA is justified. We conclude therefore that stilbene 3 molecules, which are of a rather elongated shape, behave in solution like quasispherical* particles, which allows us to calculate

* In our case, for $V=1.5 \times 10^{-21} \mathrm{~cm}^{3}$ and taking the effective length of the stilbene 3 molecule as about $2 \mathrm{~nm}$ (Fig. 1) we estimate an axial ratio of about 1:4, assuming a rod-shaped solvation shell. In contrast to this, calculations for the prolate ellipsoid of revolution based on Perrin's formulae [17] lead to an axial ratio value of about 1 which indicates that the solvation shell is effectively spherical. the effective volume $V$. The calculated volumes depended on the solvent composition. Since the solvent shell may be considered to arise from solute-solvent interaction, its volume will reflect the magnitude and/or character of this interaction.

The values of the limiting EA are constant at $r_{0}=0.375$ within the limits of experimental error. For a linear oscillator with parallel absorption and emission transition moments, the maximum value of the EA is equal 0.4. The observed difference might be due to an angle $\delta$ between absorption and emission transition moments, for which the maximum value of the EA is $r_{\mathrm{f}}=0.6 \cos ^{2} \delta-0.2$.

The value of $\delta$ obtained from the quantumchemical calculations (results in Table 2) is on the order of $1^{\circ}$, leading to $r_{\mathrm{f}}=\mathbf{0 . 3 9 9}$.

It is therefore evident that stilbene 3 in solution undergoes rapid librations which are independent of solvent composition. The average value of the mean decay time $\tau$ of total fluorescence is $1 \pm$ $0.05 \mathrm{~ns}$. This short lifetime is advantageous for laser action because, combined with the high

\begin{tabular}{llllllllr}
\hline $\begin{array}{l}\text { \% of } \\
\text { alcohol }\end{array}$ & $\begin{array}{l}T \\
{[\mathrm{~K}]}\end{array}$ & $\begin{array}{l}\text { viscosity } \\
{[\mathrm{cP}]}\end{array}$ & $\langle r\rangle$ & $\begin{array}{l}\tau^{\prime \prime} \\
{[\mathrm{ns}]}\end{array}$ & $\begin{array}{l}\boldsymbol{\tau} \\
{[\mathrm{ns}]}\end{array}$ & $\begin{array}{l}\tau^{1} \\
{[\mathrm{~ns}]}\end{array}$ & $\begin{array}{l}V \\
{\left[10^{-22} \mathrm{~cm}^{3}\right]}\end{array}$ & $\begin{array}{l}\varphi \\
{\left[10^{7} \mathrm{~s}^{-1}\right]}\end{array}$ \\
\hline 100 & 313 & 0.46 & 0.069 & 0.94 & 1.04 & 1.11 & 22 & 430 \\
100 & 293 & 0.60 & 0.092 & 0.85 & 0.95 & 1.04 & 22 & 300 \\
80 & 293 & 1.57 & 0.165 & 0.86 & 0.98 & 1.10 & 20 & 130 \\
60 & 293 & 5.02 & 0.258 & 0.89 & 1.01 & 1.13 & 16 & 50 \\
40 & 293 & 32.2 & 0.328 & 0.94 & 0.98 & 1.06 & 10 & 14 \\
20 & 293 & 82.2 & 0.358 & 0.97 & 1.02 & 1.05 & 10 & 5 \\
10 & 293 & 164.1 & 0.363 & 1.01 & 1.02 & 1.09 & 8 & 3 \\
\hline
\end{tabular}

Table 3. Parameters of thermal rotations of stilbene 3 molecules dissolved in glycerol-methanol mixtures.

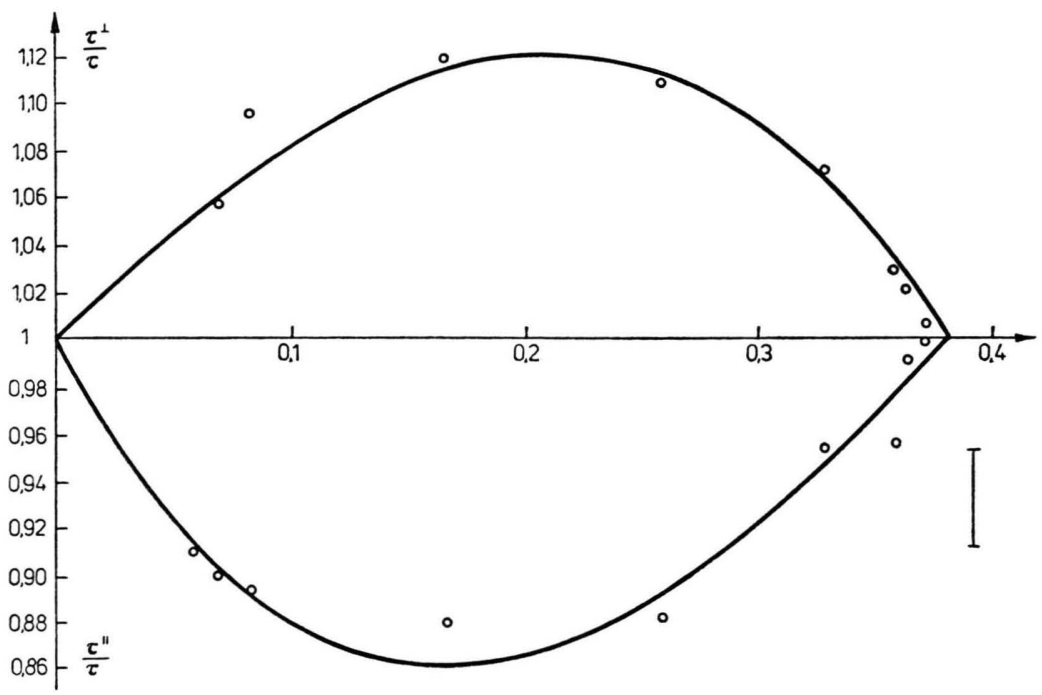

Fig. 4. Mean decay time ratios of polarized fluorescence components for stilbene 3 in mixed glycerol and methanol solvents versus EA. Solid line: values calculated for $r_{0}=0.375$. 
quantum efficiency of stilbene 3 , it implies high radiative deactivation rates and low triplet formation efficiency.

The authors would like to thank Dr. R. Raue from the Leverkusen-Bayerwerk for supplying us

[1] H. Telle, U. Brinkmann, and R. Raue, Opt. Comm. 24, 33 (1978).

[2] H. Telle, U. Brinkmann, and R. Raue, Opt. Comm. 24, 248 (1978).

[3] J. Kuhl, H. Telle, R. Schieder, and U. Brinkmann, Opt. Comm. 24, 251 (1978).

[4] R. K. Bauer and A. Kowalczyk, Opt. Comm. 23, 169 (1977).

[5] A. Warshel, J. Chem. Phys. 62, 214 (1975).

[6] P. Scharfenberg and Ch. Jung, Chem. Phys. Lett. 57, 131 (1978).

[7] F. A. Cotton and G. Wilkinson, Advanced Inorganic Chemistry, John Wiley, New York 1962.

[8] A. Kawski, I. Gryczyński, Ch. Jung, and K. H. Heckner, Z. Naturforsch. 32a, 420 (1977). with the dye studied. We also express our gratitude to Dr. R. E. Dale for careful proof reading of the manuscript.

This work was carried out as part of Research Project MR I.5.

[9] J. N. Murrell, The Theory of the Electronic Spectra of Organic Molecules, Methuen, London 1963.

[10] I. B. Berlman, Handbook of Fluorescence Spectra of Aromatic Molecules, Chapter 4, Academic Press, London 1971.

[11] O. Bastiansen, Acta Chem. Scand. 3, 408 (1949).

[12] C. J. F. Böttcher, Theory of Electric Polarization, Elsevier, Amsterdam 1952.

[13] R. L. Van Metter and R. S. Knox, Chem. Phys. 12, 333 (1976).

[14] Yu. T. Mazurenko, Opt. Spectroscopy 33, 22 (1972), 36, 491 (1974)

[15] A. Jabłoński, Z. Naturforsch. 16a, 1 (1961).

[16] R. K. Bauer, Z. Naturforsch. 18a, 718 (1963).

[17] T. Tao, Biopolymers 8, 609 (1969). 